it has become as fashionable to deny the existence of progress, as it was fashionable in the optimism of the nineteenth century to proclaim its inevitability. The truth is between the two extremes. Progress is a major fact of past evolution; but it is limited to a few selected stocks. It may continue in the future; but it is not inevitableman must work and plan if he is to achieve further progress.
Our optimism may well be tempered by reflection on the difficulties to be overcome. None the less, the demonstration of the existence of a general trend which can legitimately be called progress, and the definition of its limitations, is a fundamental contribution to thought; and we zoologists may be proud that it has been made, chiefly from the zoological side, by evolutionary biology.

\title{
Production and Technical Application of High Voltages
}

$\mathrm{T}$ HE discussion on September 14 in Section A (Mathematical and Physical Sciences) of the British Association, on the production and technical application of high voltages, was one of those selected by the Council of the Association as having a direct bearing on the life of the community. The high-voltage transmission system connecting our major power stations, and the extensive use of $\mathrm{X}$-rays in hospitals, are but two examples of the impact of high-voltage science upon society, and the extent of the applicability of this science is increasing rapidly. In no other science are physics and engineering more interdependent, and it is significant that nuclear physics would have been almost a closed book without the application of high voltages.

High voltages are familiar to us in four forms : low-frequency alternating voltages, unidirectional impulsive voltages, high-frequency alternating voltages and constant voltages. The engineer is for the most part concerned with the first three forms, the normal and the abnormal types of voltage appearing on transmission lines. The lines and the station apparatus of the 132 k.v. 'Grid' in Great Britain are tested up to two or three times normal line voltage with low-frequency alternating voltages, whilst abnormal voltagessuch as surges due to lightning or switching operations - up to ten times normal operating voltage may appear on the lines. Thus engineering laboratories equipped to supply $500-1,000$ k.v. at power-frequencies, and $1 \frac{1}{2}$ million volts from impulse generators, are necessary to test all apparatus for use on high-voltage systems, and to be in the van of immediate requirements. Constant potentials have been mainly used for radiography, X-ray therapy and physical research, and there appears to be an immediate need for voltages of the order of one million for research on nuclear physics and X-ray therapy.

There are many problems confronting the supply authorities of the 'Grid' which arise at the normal transmission voltage of 132 k.v. Among these may be mentioned the effects of corona, atmospheric pollution, gaseous discharge within the voids in so-called solid insulation, and power arc discharges initiated by atmospheric conditions and other causes, as enumerated by Mr. C. W. Marshall of the Central Electricity Board. The manufacturer is equally concerned with these problems and is constantly evolving new designs and new materials for use in high-voltage apparatus better able to withstand these destructive effects. Electrical gradients used in solid dielectrics are far lower than the theoretical permissible gradients referred to by $\mathrm{Dr}$. S. Whitehead, of the British Electrical and Allied Industries Research Association (E.R.A.), on account of the inherent fluctuations in quality of dielectrics, our lack of knowledge of the behaviour of dielectrics over a period of many years when subjected to thermal changes and mechanical forces, and also on account of the dangerously high over-voltages which are known to occur on lines. It has been estimated that these over-voltages are responsible for 75 per cent of all failures of supply systems.

As Mr. R. Davis, of the National Physical Laboratory, pointed out, studies of the behaviour of dielectrics under impulse voltages are still in their infancy, there being so many variables influencing breakdown, such as wave shape and polarity of the applied impulse, electrode shape, and medium of immersion, temperature, and number of applied impulses. Moreover, our knowledge of the nature of surges arriving at terminal stations is all too meagre: information is being obtained by cathode ray oscillographs and lightning current recorders on lines all over the world, but effective measures are not yet available to prevent damage due to lightning. In this connexion it is interesting to note that the E.R.A. has available a 11 million volt impulse generator (Fig. 1) with which to study the effects of artificially produced surges on lines and cables, this voltage being adequate to spark over the line insulation of the 
'Grid'. The effects of lightning currents of 100,000 amperes have yet to be investigated, and Prof. $\mathrm{H}$. Norinder, of Uppsala, Sweden, gave a brief account of the surge current generator and the effects of large currents on electrodes and earthing materials.

In the realm of atomic physics constant potentials are favoured, as they will produce the most stable source of high-speed ions for nuclear disintegration experiments. The five-million volt generator built by Dr. van de Graaf represents the most outstanding example of high-voltage genera-

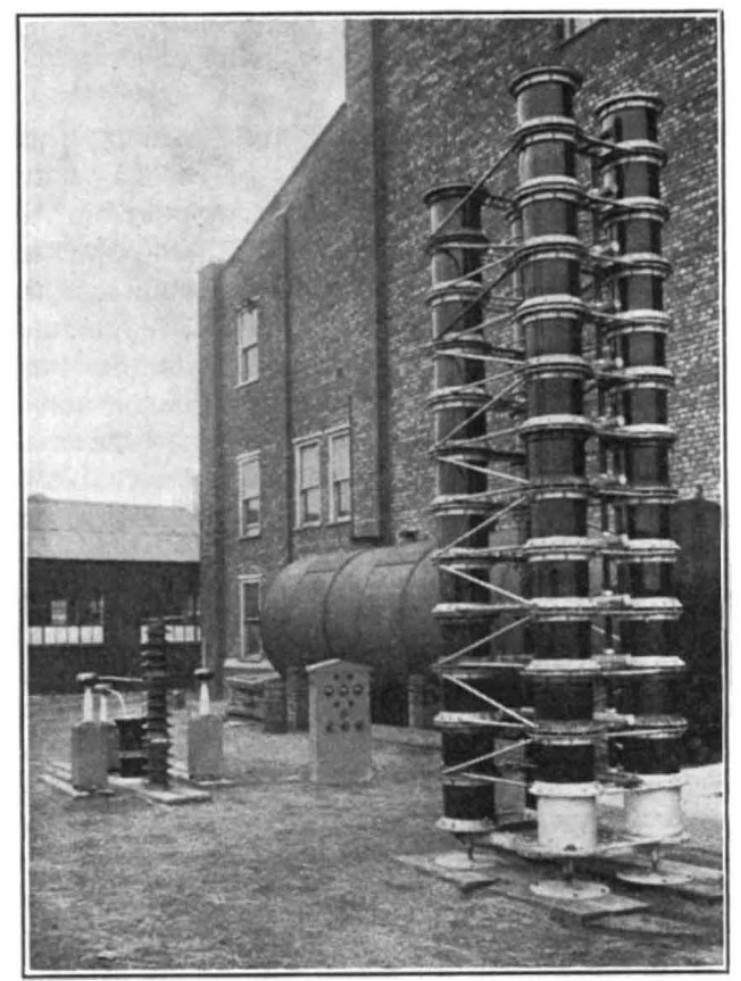

FTc. 1. $1 \frac{1}{2}$ million volt outdoor type impulse generator for use by the E.R.A. on the high voltage lines of the "Grid".

tion, but is only capable of producing one milliampere at full voltage. Thermionic valve circuits can give greater powers, but so far voltages much in excess of one million have not been produced by such means. For X-ray therapy, now so widely used in the treatment of all forms of tumours, voltages up to $250 \mathrm{k}$.v. have been used, but higher voltages are essential if the effects of short wave $\mathrm{X}$-rays are to be compared with those of the much feebler $\gamma$-rays from 5-gram bombs of radium. A number of such high-voltage $\mathrm{X}$-ray tubes is now in operation at voltages of $500-800 \mathrm{k} . \mathrm{v}$. One of the most original and successful tubes has been made by Mr. Sloan in Berkeley, California, who conceived the idea of generating a continuous high-frequency alternating voltage in the same vacuum chamber as the filament and target of the X-ray tube.

Dr. G. W. C. Kaye, of the National Physical Laboratory, described his recent researches on the measurement of $\gamma$-radiations, and announced that by using an ionization chamber large enough to absorb all the fast electrons liberated in air by the $\gamma$-rays, unification of the X-ray and $\gamma$-ray dosage measurements has been achieved. Of course, in practice it is not necessary to use a 'free air' chamber for the measurement of $\gamma$-rays or very high voltage $\mathrm{X}$-rays ; the 'free air' chamber may be replaced by a chamber having walls thick enough to absorb all the fast electrons.

An increasing use for constant voltage generators, widely affecting the community, is for the electrostatic precipitation of dust. Though a very old process of great simplicity, it has only recently been applied on a large scale for the detarring of gas, removal of poisonous fumes such as arsenic, and the elimination of dust from power-station flues. In one of the large municipal power-stations an electrostatic precipitator removes no less than 1,000 tons of dust per week which otherwise would be emitted from the chimney.

The measurement of high voltages presents a diversity of problems. Low-frequency alternating voltages may be measured by sphere spark-gap (a secondary standard) or by a crest voltmeter (a primary standard involving the measurement of the charging current of a standard condenser). The design of the condenser for one or more million volts presents the major difficulty as the condenser must be isolated from all sources of corona current. For lower voltages the condenser may be placed in a compressed gas chamber, or the new precision spark gap devised by Prof. W. M. Thornton may be used. This gap consists of parallel plates with rolled edges across which the field strength is uniform, so that sparking occurs with greater uniformity than occurs between spheres. For impulse voltages, however, this gap is not much superior to the sphere gap : an accuracy of one or two per cent is obtainable, provided that the spacing between spheres does not exceed the radius of the sphere and provided that the impulse wave front is at least one microsecond in duration. In shorter times than this, the gap measurement may be seriously in error, and the only reliable standard is the capacitance potentiometer and cathode ray oscillograph: the accuracy of measurement is then not greater than a few per cent. Constant potentials may be measured by the resistance voltmeter if sufficient power is available: for the electrostatic belt-type generator an electrostatic alternator voltmeter has been evolved which gives fair accuracy.

T. E. A. 